\title{
Variability of aerosol delivery via spacer devices in young asthmatic children in daily life
}

\author{
H.M. Janssens*, S.G. Devadason**, W.C.J. Hop ${ }^{+}$, P.N. LeSouëf**, \\ J.C. De Jongste*, H.A.W.M. Tiddens*
}

Variability of aerosol delivery via spacer devices in young asthmatic children in daily life. H.M. Janssens, S.G. Devadason, W.C.J. Hop, P.N. LeSouëf, J.C. De Jongste, H.A.W.M. Tiddens. (C) ERS Journals Ltd 1999.

ABSTRACT: Pressurized metered dose inhalers (pMDI) are widely used together with spacers for the treatment of asthma in children. However, the variability of daily medication dose for $\mathrm{pMDI} / \mathrm{spacer}$ combinations is not known. Electrostatic charge is a potential source of dose variability. Metal spacers have no static charge. This study assessed and compared within-subject variability of aerosol delivery of metal and plastic spacers. This was a randomized, crossover study in children with stable asthma aged 1-4 (group $I, n=17$ ) and 5-8 (group $I I, n=16$ ) yrs. In both groups the amount of drug delivered to the mouth by a metal spacer (Nebuchamber $\mathbb{R}$ ) and one of two

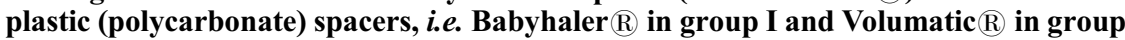
II was measured. The metal and plastic spacers were tested at home in a randomized order for 7 days each, using budesonide $(200 \mu \mathrm{g}$ b.i.d. $)$. Aerosol was collected on a filter positioned between spacer and facemask or mouth. Budesonide on the filter was assessed by high performance liquid chromatography. The mean filter dose for each child (mean \pm SD) during the 7 days was expressed as a percentage of the nominal dose. Within-subject variability was expressed as coefficient of variation (CV).

Mean filter dose in group I was $41.7 \pm 10.1 \%$ for Nebuchamber and $26.0 \pm 4.0 \%$ for Babyhaler $(p<0.001)$. Mean filter dose in group II was $50.2 \pm 9.2 \%$ for Nebuchamber and $19.4 \pm 7.2 \%$ for Volumatic $(p<0.001)$. Mean $C V$ in group I was $34 \%$ for Nebuchamber and $37 \%$ for Babyhaler $(p=0.44)$. Mean $C V$ in group II was $23 \%$ for Nebuchamber and $34 \%$ for Volumatic $(p=0.003)$.

There was substantial within-subject dose variability in aerosol delivery in children using a $\mathrm{pMDI} / \mathrm{spacer}$ at home. This variability was lower for the metal than for the plastic spacer in children 5-8 yrs of age. The dose delivered to the mouth was about two-fold higher for the metal than the plastic spacer independent of age. Eur Respir J 1999; 13: 787-791.

Pressurized metered dose inhalers (pMDI) combined with spacers are widely used for the treatment of asthma in children. Although pMDI/spacers are convenient and relatively simple to use, the dose delivery from a spacer depends on a number of patient and device features [1-4]. Predictability and reproducibility of dosing are relevant for any drug therapy. This general pharmacological principle allows the clinician to select the proper dose and device for a given patient. Considerable between-subject variability in aerosol delivery from spacers has been described $[1,5]$. These results were based on only one or two observations per child in a laboratory setting, recorded within a short interval. Though such studies may provide a good impression of the quality of the different devices, it may not adequately represent the situation in daily life, which is obviously important when prescribing inhaled drugs.

One of the factors that can contribute to variability in aerosol delivery is the material from which the spacer is made. Until recently, spacers were made of plastic. Various studies have shown that electrostatic charge on plastic spacers decreases drug delivery $[6,7]$. Recently, a metal spacer (Nebuchamber $\mathbb{R}$ (Astra Draco, Lund, Sweden)) has
*Dept of Paediatrics, Division of Paediatric Respiratory Medicine, ${ }^{+}$Dept of Biostatistics, Sophia Children's Hospital, and Erasmus Medical Centre, Rotterdam, The Netherlands. **Dept of Respiratory Medicine, Princess Margaret Hospital for Children, Perth, Australia

\section{Correspondence: H.M. Janssens}

Dept of Paediatric Respiratory Medicine Sophia Children's Hospital

PO box 2060, 3000 CB Rotterdam

The Netherlands

Fax: 31104636772

\section{Keywords: Aerosol}

asthma

budesonide

children

electrostatic charge

spacer

Received: February 261998

Accepted after revision November 181998

This study was supported by a grant from Astra The Netherlands. been developed that does not have this disadvantage [5]. The aim of this study was to assess within-subject variability of aerosol delivery from several spacers in asthmatic children 1-8 yrs old in a daily life setting in an open randomized, crossover study. Secondary aims were to compare the variability of aerosol delivery from metal and plastic spacers and to investigate whether within-subject variability was age dependent.

\section{Materials and methods}

\section{Study population}

Children aged 1-8 yrs with stable asthma and on daily inhalation therapy were recruited from the asthmatic population treated at the Princess Margaret Hospital for Children in Perth, Australia. Stable asthma was defined as having no exacerbations requiring additional oral corticosteroids or any change in asthma medication for at least one month prior to the onset of the study. None of the children suffered from any other disorder that could affect 
lung function. The children were divided into two age groups, 1-4 yrs (group I) and 5-8 yrs (group II), to distinguish between inhalation via a face mask or a mouthpiece. Written informed consent was obtained from all parents. The study was approved by the local ethics committee.

\section{Materials}

Group I used the metal Nebuchamber $\mathbb{R})(250 \mathrm{~mL}$; Astra) and the polycarbonate Babyhaler $\mathbb{R}$ [8] (350 mL; Glaxo Wellcome, London, UK) both with face masks. Group II used the Nebuchamber(R without face mask and the polycarbonate Volumatic $\mathbb{R})(750 \mathrm{~mL}$; Glaxo Wellcome). The inlet of the Babyhaler was slightly adapted to obtain a good fit and a straight plume of the Pulmicort $\mathbb{R}$ pMDI (Astra) into the spacer. For the Volumatic a small plastic connector (Astra) was used to fit the Pulmicort pMDI. The day before the spacers were allocated to the children, they were washed in water with normal household detergent, rinsed thoroughly with warm water and drip-dried according to the instructions for use from the manufacturers. Each week the subjects received a clean spacer. Budesonide pMDI $200 \mu \mathrm{g} \cdot \operatorname{dose}^{-1}$ (Pulmicort $\mathbb{R}$ ) was used as the study medication. Each child received a new budesonide pMDI. The first 10 actuations of a new pMDI were wasted to avoid the variable doses in the first 10 actuations $[9,10]$. Aerosol from the spacers was collected on a filter (Vital Signs, Totowa, NJ, USA) inserted between the face mask or the patient's mouth and spacer. The filter has been shown to retain $>99 \%$ of the budesonide delivered from a spacer [5]. The filter added a dead space of $20 \mathrm{~mL}$ to the system. The pressure drop over the filter was $230 \mathrm{~Pa}$ at $60 \mathrm{~L} \cdot \mathrm{min}^{-1}$ [5], which is approximately one fifth of the airway resistance of a young child [11].

To evaluate asthma stability, all parents filled out a diary card on asthma symptoms twice a day during the study period. The symptoms cough, wheeze and shortness of breath, and cooperation during drug administration were each assigned a score of $0-3$.

\section{Study procedure}

The study was designed as a randomized, crossover study. During the 3 study weeks, the investigator visited the children 4-times at home. On the first visit the use of the $\mathrm{pMDI} / \mathrm{spacers}$ with the filter was demonstrated and study materials provided. The study medication had to be administered twice daily, before regular maintenance therapy, which was continued during the study period using the patient's own medication and device. Before each administration the parents attached a new filter to the spacer. The spacer was held in a horizontal position, while ensuring a close fit of the face mask or lips sealed around the mouthpiece with the child in an upright position. The pMDI had to be shaken vigorously for $10 \mathrm{~s}$ just prior to actuation. One puff of budesonide was actuated into the spacer. The child had to inhale for $60 \mathrm{~s}$ with quiet tidal breathing. Subsequently, the filter was removed from the spacer and both sides of the filter holder were sealed with sticky tape. Each filter was labelled with a unique code. Finally, the filters were wrapped in aluminium foil to protect the budesonide from destabilization by light. In the first study week, all children practised the use of both spacers with filters and a placebo. After this run-in period the children were randomized to start with budesonide via the metal or the plastic spacer. On the second visit any problems were discussed. The administration procedure was demonstrated by the child, corrected if necessary, and new spacers, filters and pMDI were provided. On the third visit the filters and the first spacer were collected and the second spacer and set of filters were issued. On the fourth visit the filters and the second spacer were collected. Thus, for each spacer 14 samples were obtained per child, i.e. two samples per day.

\section{Filter analysis}

All filters were analysed in a blinded fashion. Filter and filter holder were washed in ethanol containing an internal standard (fluocinolone acetonide). Budesonide was quantified by high-performance liquid chromatography (HPLC), using an ethanol-water (43:57) mobile phase and a Supelcosil LC-18 column $(5 \mu \mathrm{m}$ particles, $5 \times 0.46 \mathrm{~cm}$ ID; Supelco, Bellafonte, PA, USA). Budesonide was detected by UV spectrophotometry at a wavelength of $254 \mathrm{~nm}$.

\section{Statistical analysis}

SPSS for Windows version 6.1 (SPSS, Chicago, IL, USA) was used for the statistical analysis. The filter dose, i.e. the amount of budesonide deposited on the filter, was expressed as a percentage of the nominal dose. The mean \pm SD of the filter dose of the 14 samples collected in 1 week was calculated for each child. Within-subject variability was expressed as coefficient of variation (CV). Paired ttests were used to compare means, after verifying that there were no period or carry-over effects [12]. The presence of a priming effect was investigated by plotting all filter doses for each child against the 14 consecutive sample numbers and drawing individual regression lines through these data points. The mean slope of the individual regression lines per spacer was calculated for the two groups.

Relationships between age and filter doses, age and within-subject $\mathrm{CV}$, between individual filter doses of both spacers and between within-subject $\mathrm{CV}$ of both spacers were calculated by means of regression analysis. Mean asthma scores, defined as the sum of asthma symptoms recorded on the diary card during one week, while using one of the two spacers were compared by paired t-test.

This analysis was repeated after excluding the samples where the child had not cooperated (score 2 and 3 ) during the procedure, as indicated in the diary. A p-value $\leq 0.05$ (two-sided) was considered significant.

\section{Results}

Forty-one children were enrolled into the study. Eight dropped out after the run-in period. The reasons for drop out were noncooperative child $(n=4)$, asthma exacerbation $(\mathrm{n}=1)$ and noncompliant parents $(\mathrm{n}=3)$. Seventeen children (12 male) in group I, and 16 (12 male) in group II 
completed the study. Mean age in group I was 40 months (range 17-59), mean age in group II was 83 months (range 65-104). All children had been using a pMDI/spacer for more than 6 months, except one who had been using a drypowder device. The devices used before study entry were for group I: Breath-a-Tech $\mathbb{R}$ (Scott Dibben, New Castle, NSW, Australia) $(n=8)$, Volumatic $(n=2)$, Aerochamber $\mathbb{R}$ (Trudell Medicals, London, Ontario, Canada) $(n=6)$, Babyhaler $(n=1)$, and for group II: Breath-a-Tech $\mathbb{R}(n=3)$, Volumatic $(\mathrm{n}=12)$, Turbuhalerß $(\mathrm{n}=1)$ (Astra).

\section{Filter dose}

Filter doses (mean $\pm \mathrm{SD})$ for the spacers in group I and II are shown in figure 1 and in table 1 . Filter doses were significantly higher $(\mathrm{p}<0.0001)$ in the Nebuchamber than in the plastic spacers. There was no significant correlation between age and filter dose in group I and II with both spacers. In group II there was a significant positive correlation between the filter dose for the Nebuchamber and the Volumatic $(\mathrm{r}=0.79, \mathrm{p}=0.0003)$. In other words, children with higher filter doses for the Nebuchamber also
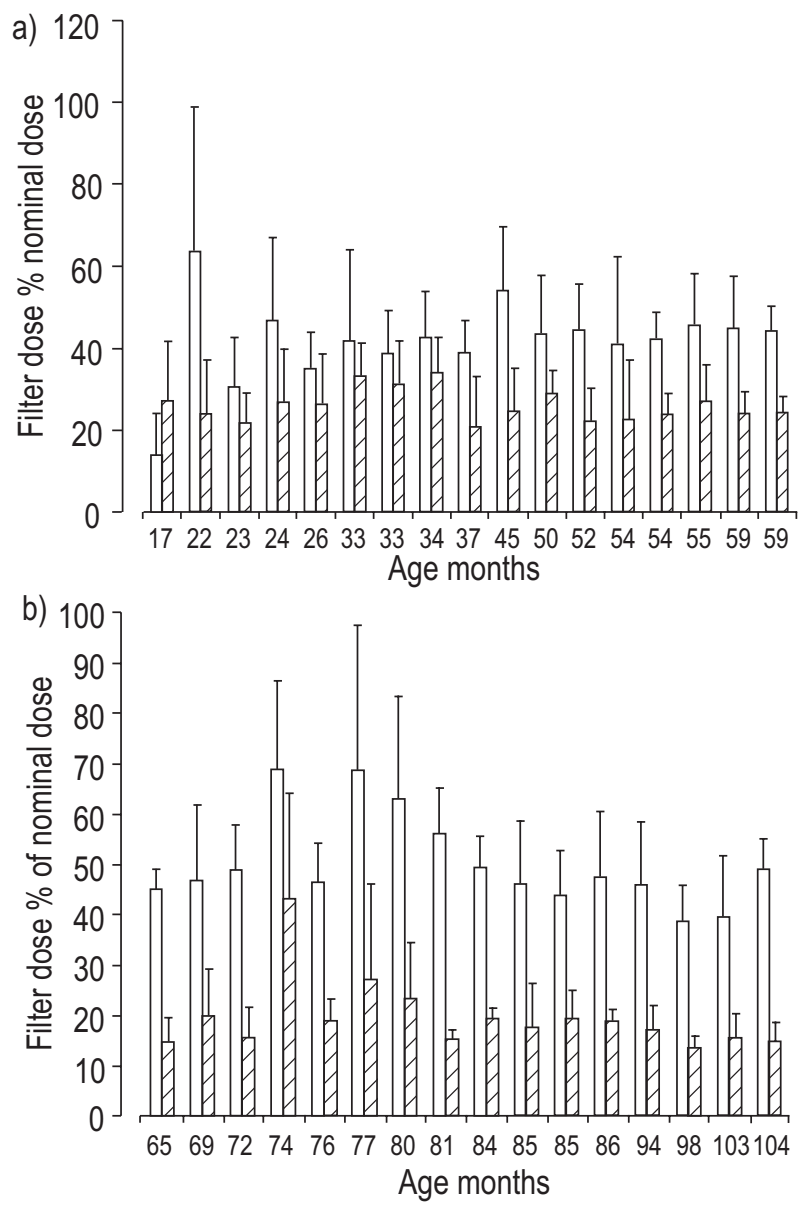

Fig. 1. - Aerosol dose delivered to the mouth for various spacer devices in relation to age. a) Group I (age $1-4$ yrs, $n=17$ ) with mean ( \pm SD) filterdose of budesonide for each child for the Nebuchamber $(\square)$ and the Babyhaler $(\mathbb{Z})$ as a percentage of the nominal dose $(200 \mu \mathrm{g})$. b) Group II (age 5-8 yrs, $n=16$ ) with mean $( \pm$ SD) filter-dose of budesonide for each child for the Nebuchamber $(\square)$ and the Volumatic $(\mathbb{Z})$ as a percentage of the nominal dose $(200 \mu \mathrm{g})$. Each pair of columns represents one child in ascending order of age.
Table 1. - Mean filter dose and within-subject variability in aerosol delivery

\begin{tabular}{|c|c|c|c|c|}
\hline & \multicolumn{2}{|c|}{$\begin{array}{c}\text { Group I } \\
\text { (1-4 yrs) }\end{array}$} & \multicolumn{2}{|c|}{$\begin{array}{l}\text { Group II } \\
\text { (5-8 yrs) }\end{array}$} \\
\hline & $\begin{array}{l}\text { Nebu- } \\
\text { chamber }\end{array}$ & Babyhaler & $\begin{array}{l}\text { Nebu- } \\
\text { chamber }\end{array}$ & Volumatic \\
\hline Filter dose & $41.7 \pm 10.1$ & $26.0 \pm 4.0$ & $50.3 \pm 9.2$ & $19.4 \pm 7.2^{\ddagger}$ \\
\hline $\begin{array}{l}\text { Within- } \\
\text { subject } \mathrm{CV}^{\dagger}\end{array}$ & $34.1 \pm 15.6$ & $37.2 \pm 5.0^{\S}$ & $23.1 \pm 9.1$ & $34.0 \pm 6.5^{\S}$ \\
\hline
\end{tabular}

Results are mean $\pm \mathrm{SD}$ in $\%$ of nominal dose. ${ }^{\dagger}$ : coefficient of variation $(\mathrm{CV})$ as measure of variability. $\star_{\mathrm{w}} \mathrm{p}<0.0001$ compared with Nebuchamber; ${ }^{\S}: \mathrm{p}=0.44$ compared with Nebuchamber; ${ }^{\$}$ : $\mathrm{p}=0.003$ compared with Nebuchamber.

tended to have higher filter doses for the Volumatic. This correlation was not found in group I.

In group I, a small but significant priming effect was found for the Babyhaler but not for the Nebuchamber $(p<0.05)$. The filter dose in the Babyhaler increased $0.4 \%$ per consecutive sample, or $0.8 \%$ per day. In group II there was no correlation between filter dose and sample number for the Nebuchamber or for the Volumatic.

\section{Variability}

Within-subject variability of aerosol delivery in both groups (mean $\pm \mathrm{SD})$ is shown in table 1 . In group I the within-subject CV for the Nebuchamber and for the Babyhaler were similar $(\mathrm{p}=0.44)$. In group II the within-subject $\mathrm{CV}$ for the Nebuchamber was significantly smaller than for the Volumatic $(\mathrm{p}=0.003)$. The range of within-subject $\mathrm{CV}$ was large in both groups for all spacers. In group II, children with a higher within-subject CV for the Nebuchamber also had a higher within-subject $\mathrm{CV}$ for the Volumatic $(r=0.7, p=0.028)$. This correlation was not found in group I. The within-subject CV decreased significantly with age in group I for the Nebuchamber (figure 2). For the Babyhaler, within-subject $\mathrm{CV}$ tended to decrease with age $(\mathrm{r}=-0.5, \mathrm{p}=0.06)$, but this was not the case for the spacers in group II.

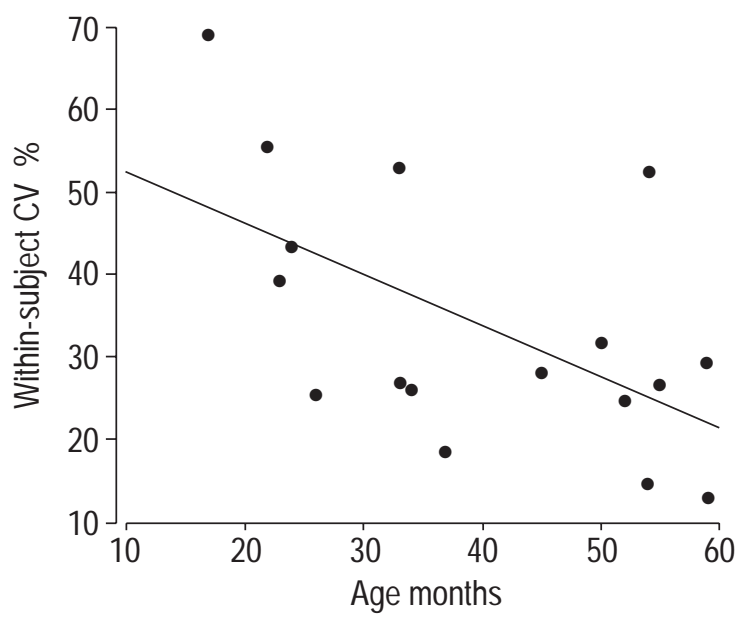

Fig. 2. - Correlation between within-subject variability (coefficient of variation $(\mathrm{CV}))$ of dose and age for the Nebuchamber in group $\mathrm{I}$. ( $\mathrm{r}=-0.6$, $\mathrm{p}=0.02$.) 


\section{Diary cards}

Mean asthma scores were similar during the use of the different spacers in both groups. The majority of children were cooperative (score 0 and 1) during the procedure. Of the 476 assessments in group I, scores 2 or 3 were recorded 41 times $(8.6 \%)$ and of the 448 assessments in group II, twice $(0.4 \%)$. The analysis of the data was repeated after excluding the assessments where the cooperation was scored as 2 or 3 . This did not change the outcomes.

\section{Daily life observations}

Despite careful repeated instruction, mistakes were still made. Some were mentioned on the diary cards or could be concluded from the raw data, while others were observed. Recorded faults were not shaking the pMDI before actuation, leaving the cap on the pMDI during actuation, the wrong number of actuations (6 samples of $>100 \%$ and 15 samples of $>80 \%$ of the nominal dose), and using the face mask of the Nebuchamber upside-down by a 17month-old child, resulting in a very low filter dose.

\section{Discussion}

This study assessed the within-subject variability in aerosol delivery from $\mathrm{pMDI} / \mathrm{spacer}$ devices in asthmatic children 1-8 yrs old in a daily life situation. It was found that the within-subject variability in aerosol delivery was considerable. Former studies have already indicated large between-subject variability in aerosol delivery in children using plastic and metal spacers [1, 5, 13]. In clinical practice the performance of inhalation devices should be known in order to prescribe medication dosages correctly and consistently.

There are several sources of dose variability. Electrostatic charge which retains the drug in the spacer can build up on plastic (polycarbonate) $[14,15]$. Therefore, it was hypothesized that electrostatic charge on plastic spacers is a potential source of dose variability. It was found that the within-subject variability in the metal and plastic spacers was the same in younger children, and significantly less in the metal spacer in older children. A small but significant priming effect was found in the Babyhaler but not in the Volumatic. Previously, it has been shown that several actuations from a pMDI reduce the negative effect of electrostatic charge on aerosol delivery[5]. The present results indicate that electrostatic charge probably plays a minor role as a cause of variability. The fact that no priming effect was shown in the Volumatic is difficult to interpret. It is possible that some priming was present but other factors, such as valve design or volume, in the Volumatic were more important for aerosol delivery and therefore masked a possible small effect of priming. It is also possible that because of the larger volume, and therefore larger inner surface, the priming was not sufficient to show an increase of filter dose within a week of use.

It was found that within-subject variability of aerosol delivery was inversely related to age only in children $<5$ yrs old. This relation was stronger for the Nebuchamber than for the Babyhaler. A difference between age groups might be explained by the use of a face mask in group I.
The effectiveness of a face mask depends on the fit and the cooperation of the child. A fitting problem with the facemask can lead to aerosol dilution by air entrained from the side of the facemask. The low mean filter dose of the 17month old child, who used the face mask of the Nebuchamber upside-down, illustrates this possibility. However, mask fit was not systematically investigated in this study, so its importance can only be speculated upon.

Other patient-related factors could have contributed to variability in aerosol delivery. Within-subject variability for the Nebuchamber and the Volumatic in group II were positively correlated. A likely explanation for this correlation is that consistent differences in inhalation technique or administration procedure between children occurred. This is confirmed by the positive correlation between the mean filter dose for the Nebuchamber and the Volumatic, which indicates that children with a better technique are able to inhale more aerosol from each device. Mistakes were made despite repeated instruction: filter doses of $0 \%$ and $>100 \%$ demonstrated that the instructions had not been followed correctly by some children and parents. These data were not excluded from the analysis because mistakes in the administration procedure will always contribute to variability in aerosol delivery in daily life. In an in vitro study, it has been shown that the way in which the pMDI is handled before inhalation contributes to the variability of dose. For instance, not shaking the pMDI reduced the dose delivered by $25.5 \%$ [16]. The present results and observations confirm that differences in inhalation technique and administration procedure are indeed important factors in the variability in aerosol delivery in daily life.

It can be argued that the plastic spacers were not designed for the use of a Pulmicort pMDI. Each formulation/spacer combination may behave differently, therefore it is not known how the results would have varied if a different drug had been used. However, the large withinsubject variability appeared to be not only spacer-dependent and can therefore be considered as a general feature in the use of $\mathrm{pMDI} /$ spacers for inhalation therapy in young children. Further research to assess dose variability in other $\mathrm{pMDI} / \mathrm{spacer}$ combinations is needed.

Within-subject variability is expressed as $\mathrm{CV}$ which cannot be interpreted separately from the mean filter dose. There were large differences between the filter doses of the various spacers, whereas the variabilities expressed as $\mathrm{CV}$ were similar. This indicates that the plastic spacers with the lower dose also had the lowest absolute variability. It is encouraging that the average amount of aerosol a child received over a 1 -week period appeared reasonable. The filter dose was significantly less with the two plastic spacers than with the metal Nebuchamber. It is likely that the electrostatic charge on the plastic spacers contributed to this difference, as it has been shown that the Babyhaler performs as well as a Nebuchamber when electrostatic charge is minimized by detergent coating [17]. However, this was not the case in the present study, as the majority of plastic spacers are actually used without coating, in accord with the manufacturer's instructions.

In this study, filter dose was found to be independent of age in both groups. This is in agreement with previous studies $[1,13,17]$. The difference in mean filter dose between group I and II is probably not an effect of age, but a result of the use of a face mask in group I. The face mask 
adds dead space and may cause air entrainment, thereby reducing the filter dose in group I selectively.

It can be argued that filter studies only provide information about the amount of aerosol delivered to a patient and not about deposition in the respiratory tract. In the present study, the primary aim was to assess the reproducibility of aerosol delivery from $\mathrm{pMDI} /$ spacer combinations in children and not to assess the efficiency of inhalation systems to deliver aerosol to the lungs. Therefore, the assessment of aerosol delivery by means of a filter is a valid method to obtain such information. Clearly, further research should focus on the therapeutic implications of the present findings.

In conclusion, there was a considerable within-subject variability in aerosol delivery from both metal and plastic spacers in asthmatic children 1-8 yrs old in daily life. This variation was age dependent below the age of 4 yrs. Only a small part of the variability in aerosol delivery could be attributed to differences in spacers. The absence of electrostatic charge on the metal spacer did not result in more constant dose delivery in children aged 1-4 yrs. However, the relative within-subject variability was less for metal than for plastic spacers in children $>5$ yrs old. Factors such as spacer design, use of a face mask, age, inhalation technique and compliance with a correct administration procedure are possible causes of variability in aerosol delivery. Further research is required to investigate the causes and consequences of dose variability in aerosol treatment with pressurized metered dose inhalers/spacers in asthmatic children.

\footnotetext{
Acknowledgements. The authors would like to thank E. Berg and C. Rossborg from Astra Draco, Lund, Sweden for the analysis of the filters. The parents and children are acknowledged for their participation in the study.
}

\section{References}

1. Agertoft L, Pedersen S. Influence of spacer device on drug delivery to young children with asthma. Arch Dis Child 1994; 71: 217-219.

2. Barry $\mathrm{PW}, \mathrm{O}^{\prime}$ Callaghan C. Inhalational drug delivery from seven different spacer devices. Thorax 1996; 51: 835-840.

3. Everard ML, Clark AR, Milner AD. Drug delivery from holding chambers with attached facemask. Arch Dis Child 1992; 67: 580-585.

4. Tal A, Golan H, Grauer N, Aviram M, Albin D, Quastel MR. Deposition pattern of radiolabeled salbutamol inhal- ed from a metered-dose inhaler by means of a spacer with mask in young children with airway obstruction. $J$ Pediatr 1996; 128: 479-484.

5. Bisgaard H, Anhoj J, Klug B, Berg E. A non-electrostatic spacer for aerosol delivery. Arch Dis Child 1995; 73: 226-230.

6. Barry PW, O'Callaghan C. The effect of delay, multiple actuations and spacer static charge on the in vitro delivery of budesonide from the Nebuhaler. Br J Clin Pharmacol 1995; 40: 76-78.

7. Wildhaber JH, Devadason SG, Hayden MJ, et al. Electrostatic charge on a plastic spacer device influences the delivery of salbutamol. Eur Respir $J$ 1996; 9: 1943-1946.

8. Kraemer R, Birrer P, Modelska K, Aebischer CC, Schöni MH. A new baby-spacer device for aerosolized bronchodilator administration in infants with bronchopulmonary disease. Eur J Pediatr 1992; 151: 57-60.

9. $\quad$ Berg E. In vitro properties of pressurized metered dose inhalers with and without spacer devices. J Aerosol Med 1995; 8: S3-10.

10. Cyr T, Graham SJ, Li RKY, Lovering EG. Low first-spray drug content in albuterol metered-dose inhalers. Pharm Res 1991; 8: 658-660.

11. Stocks J, Marchal F, Kraemer R, Gutkowski P, Yishay E, Godfrey S. Plethysmographic assessment of functional residual capacity and airway resistance. In: Stocks J, Sly P, Tepper R, Morgan W, eds. Infant Respiratory Function Testing. New York, Wiley-Liss Inc., 1996; p. 232.

12. Hills M, Armitage P. The two-period cross-over clinical trial. Br J Clin Pharmacol 1979; 8: 7-20.

13. Bisgaard H. A metal aerosol holding chamber devised for young children with asthma. Eur Respir J 1995; 8: 856860.

14. Wildhaber JH, Devadason SG, Eber E, et al. Effect of electrostatic charge, flow, delay and multiple actuations on the in vitro delivery of salbutamol from different small volume spacers for infants. Thorax 1996; 51: 985-988.

15. Barry PW, O'Callaghan C. Multiple actuations of salbutamol MDI into a spacer device reduce the amount of drug recovered in the respirable range. Eur Respir J 1994; 7: $1707-1709$.

16. Everard ML, Devadason SG, Summers QA, LeSouëf PN. Factors affecting total and "respirable" dose delivered by a salbutamol metered dose inhaler. Thorax 1995; 50: 746749.

17. Wildhaber JH, Devadason SG, Hayden MJ, Eber E, Summers QA, LeSouëf PN. Aerosol delivery to wheezy infants: comparison between a nebulizer and two small volume spacers. Pediatric Pulmonology 1997; 23: 212 216. 